\title{
Insufficient Resolution Response in the Hippocampus of a Senescence-Accelerated Mouse Model — SAMP8
}

\author{
Xiuzhe Wang • Elena Puerta • Angel Cedazo-Minguez • \\ Erik Hjorth • Marianne Schultzberg
}

Received: 5 May 2014 / Accepted: 28 May 2014 /Published online: 10 June 2014

(C) The Author(s) 2014. This article is published with open access at Springerlink.com

\begin{abstract}
Aging is the primary risk factor for Alzheimer's disease (AD), and it is known that inflammation is associated with both aging and $\mathrm{AD}$. To resolve inflammation, biosynthesis of the specialized pro-resolving mediators (SPMs) is enhanced in a programmed and active manner. We investigated the effect of age on resolution by analyzing hippocampal tissue from 2- and 9-month-old senescence-accelerated mouse prone 8 (SAMP8), as well as age-matched senescenceaccelerated mouse resistant 1 (SAMR1). Pro-inflammatory markers increased upon age in SAMP8 mice and were also higher than those in age-matched SAMR1 mice. However, neither SPMs nor their receptors were enhanced upon age in SAMP8 mice compared to age-matched SAMR1 mice. Analysis of SPM biosynthetic enzymes revealed elevated levels of leukocyte type 12-lipoxygenase (L12-LOX) and decreased 5LOX levels upon age in SAMR1 mice, whereas they remained unchanged in SAMP8 mice. Moreover, we found partial colocalization of L12-LOX and amyloid beta $(\mathrm{A} \beta$ ) staining, as well as correlation between L12-LOX and phosphorylated tau levels in SAMP8, but not SAMR1 mice. Thus, we conclude that the resolution response in SAMP8 mice is insufficient to counteract the increased inflammation with age, and this may have a role in the development of AD-like pathologies.
\end{abstract}

X. Wang $\cdot$ E. Hjorth $\cdot$ M. Schultzberg $(\bowtie)$

Department of Neurobiology, Care Sciences and Society, Section of Neurodegeneration, Karolinska Institutet, 14186 Stockholm, Sweden

e-mail: Marianne.Schultzberg@ki.se

E. Puerta $\cdot$ A. Cedazo-Minguez

Department of Neurobiology, Care Sciences and Society, Section of

Alzheimer's Disease Research Center, Karolinska Institutet,

Stockholm, Sweden

E. Puerta

Department of Pharmacology, School of Pharmacy, University of

Navarra, Pamplona, Spain
Keywords Aging · Alzheimer $\cdot$ Lipoxygenase $\cdot \mathrm{LXA}_{4}$. Resolution of inflammation $\cdot$ RvD1 $\cdot$ Tau

\section{Introduction}

Increased proportion of aged individuals is a global phenomenon, raising concerns about age-related diseases, including Alzheimer's disease (AD). AD is the most common type of dementia and a progressive neurodegenerative disease with no cure up to date. The etiology of AD is still not clear, despite the fact that the two pathological hallmarks, increased senile plaques consisting of amyloid $\beta(A \beta)$ and neurofibrillary tangles composed of hyperphosphorylated tau (P-tau), have been known for over 100 years. While genetic factors that promote disease development have been identified, age is the primary risk factor for $\mathrm{AD}$ (Kawas et al. 2000).

Inflammation is known to be increased in aging, as indicated by increased levels of serum pro-inflammatory cytokines, such as interleukin (IL)-6 and tumor necrosis factor- $\alpha$ (TNF- $\alpha$ ) (Wei et al. 1992; de Gonzalo-Calvo et al. 2010), and activation of glial cells in the brain (Ogura et al. 1994; Sheffield and Berman 1998; Campuzano et al. 2009). In $\mathrm{AD}$, the age-related changes are even more pronounced (McGeer et al. 1987; Carpenter et al. 1993; Swardfager et al. 2010; Serrano-Pozo et al. 2011). There is ample evidence suggesting the pathogenic role of inflammation in AD. Long-term consumption of nonsteroidal anti-inflammatory drugs (NSAIDs) was shown to associate with lower prevalence of AD (McGeer et al. 1990; Stewart et al. 1997, in't Veld et al. 2001). In AD animal models, by ablating or blocking p40, a subunit of the pro-inflammatory cytokines IL-12 and IL-23, AD-like pathologies and cognitive impairment were reduced (Vom Berg et al. 2012). Moreover, inflammation has been shown to regulate the processing of amyloid precursor protein (APP) and, thus, influences $A \beta$ production (Sastre 
et al. 2008). Hence, it is reasonable to suspect a role of inflammation in the pathogenesis of AD. The exaggerated increase of inflammation in the $\mathrm{AD}$ brain suggests that $\mathrm{AD}$ could be a result of abnormal aging of the brain (Herrup 2010).

It is not until recent years that it became clear that inflammation is balanced by a programmed active process termed resolution (Serhan 2010), in which activation of inflammatory cells is reduced, levels of proinflammatory cytokines are downregulated, antiinflammatory cytokines are upregulated, the tissue is healed, and homeostasis is restored (Serhan 2011). Resolution is mediated by specialized pro-resolving mediators (SPMs), including lipoxins (LXs), resolvins (Rvs), protectins/neuroprotectins (PDs/NPDs), and maresins (MaRs) (Serhan 2010). Biosynthesis of SPMs involves sequential oxidization of polyunsaturated fatty acids (PUFAs) by lipoxygenases (LOXs) and/or cyclooxygenase (COX), including leukocyte type 12-LOX (L12LOX, mouse homologue to human 15-LOX-1) and 5LOX (Recchiuti and Serhan 2012). In a skin inflammation model, the increased biosynthesis of $\mathrm{LXA}_{4}$ in the inflammation site shortly after the peak of proinflammatory activities demonstrates a typical programmed resolution response to inflammation (Levy et al. 2001). By activating their receptors, SPMs initiate and promote resolution of inflammation (Serhan et al. 2011; Bannenberg and Serhan 2010). The LXA receptor/formyl peptide receptor 2 (ALX/FPR2, also named as FPR2, ALX, or FPR2/ALX) is the receptor for both $\mathrm{LXA}_{4}$ and RvD1 (Fiore et al. 1994; Krishnamoorthy et al. 2012), and the chemokine receptor 23 (ChemR23) is the receptor for RvE1 (Arita et al. 2005) and a chemoattractant protein, chemerin (Wittamer et al. 2003). Both of these SPM receptors are $\mathrm{G}$ protein-coupled receptors.

Failure of resolution may lead to chronic inflammation and continuous tissue destruction, which may finally cause tissue dysfunction (Serhan 2007). Since both AD and "healthy aging" without dementia have signs of elevated inflammation, a dividing line could be an impaired resolution response specific for $\mathrm{AD}$. To date, it is not known how resolution of inflammation in the brain is affected by aging. In the present study, we have analyzed the major markers for resolution in 2- and 9month-old senescence-accelerated mouse prone 8 (SAMP8), a mouse model for accelerated aging with AD-like pathology and symptoms (Pallas et al. 2008). Age-matched animals of the senescence-accelerated mouse resistant 1 (SAMR1), which have a relatively normal aging process with intact cognitive function, were used as controls. SAMP8 and SAMR1 mice are selected by phenotype difference from one common background strain, AKR/J mice (Takeda et al. 1981; Miyamoto et al. 1986). By comparing these two different aging models, we tested the hypothesis that an abnormal aging process related to $\mathrm{AD}$ displays insufficiency in the mechanisms governing resolution of inflammation.

\section{Materials and Methods}

Animals

Male SAMP8 and SAMR1 mice were purchased from Harlan Laboratories (Barcelona, Spain). The mice were housed in a humidity- and temperature-controlled environment with a 12:12-h light-dark cycle. The animals had access to food and water ad libitum. The usage of animals in this study was approved by the Ethics Committee of the University of Navarra.

Brain tissues from 2- and 9-month-old SAMP8 and SAMR1 mice were analyzed with regard to factors involved in the resolution of inflammation by enzyme immunoassay (EIA), western blotting, and immunohistochemistry. Animals used for the biochemical assays were euthanized by intraperitoneal (i.p.) injection of a lethal dose of pentobarbital sodium (150 mg/kg body weight). The brains were dissected immediately, and the hippocampi from the left and right hemispheres were taken for EIA and western blotting, respectively. The tissues were kept at $-80{ }^{\circ} \mathrm{C}$ until further analysis. Animals employed for morphology were anesthetized with i.p. injection of pentobarbital sodium ( $50 \mathrm{mg} / \mathrm{kg}$ body weight) and perfused with $4 \%$ paraformaldehyde (PF) solution through the left ventricle. The brains were dissected immediately after perfusion, post-fixed in $4 \% \mathrm{PF}$ at $4{ }^{\circ} \mathrm{C}$ overnight, and subsequently soaked in $10 \%$ sucrose at $4{ }^{\circ} \mathrm{C}$ until further processing.

\section{Enzyme Immunoassay}

The $\mathrm{LXA}_{4}$ enzyme immunoassay (EIA) kit (Oxford Biochemical Research, MI, USA) and the resolvin D1 (RvD1) EIA kit (Cayman Chemical, Ann Arbor, USA) were used according to the manufacturers' instructions for the analysis of extracts of the hippocampus from 2- and 9-month-old SAMP8 mice (both $n=5), 2$-month-old SAMR1 mice $(n=4)$, and 9-monthold SAMR1 mice $(n=5)$. Briefly, the hippocampus from the left hemisphere was homogenized in ethanol and centrifuged at $1,500 \mathrm{~g}$ for $15 \mathrm{~min}$. The supernatants were diluted by UltraPure water (Cayman Chemical, Ann Arbor, USA) and acidified to final $\mathrm{pH} 3.5$. The acidified samples were added to a methanol preconditioned $\mathrm{C} 18$ column (Waters Corporation, MA, USA), and the column was subsequently washed by water and hexane. The SPMs were eluted by adding methyl formate to the column. Eluted solution was evaporated by nitrogen gas, and finally the extracted samples were 
resuspended by the extraction buffer supplied with the $\mathrm{LXA}_{4}$ EIA kit.

\section{Western Blotting}

The hippocampus from the right hemisphere ( $n=5$ animals per group) was homogenized by sonication in a homogenization buffer containing $20 \mathrm{mM}$ Tris- $\mathrm{HCl}$, pH $6.8,137 \mathrm{mM} \mathrm{NaCl}$, $2 \mathrm{mM}$ EDTA, $0.5 \mathrm{mM}$ IBMX, $2 \mathrm{nM}$ okadaic acid, $1 \%$ protease inhibitor cocktail (Sigma-Aldrich Co., Saint Louis, USA), and phosphatase inhibitors (Halt ${ }^{\mathrm{TM}}$ cocktail, PierceBradford, IL, USA). The homogenates were centrifuged at $20,000 \mathrm{~g}$ for $15 \mathrm{~min}$ at $4{ }^{\circ} \mathrm{C}$, and the supernatants collected for analysis. Protein determination was performed by a bicinchoninic acid kit (Sigma-Aldrich Co., Saint Louis, USA). Samples containing $40 \mu \mathrm{g}$ protein each were mixed with an equal volume of $2 \times$ Laemmli buffer (Sigma-Aldrich Co., Saint Louis, USA) and boiled at $95{ }^{\circ} \mathrm{C}$ for $5 \mathrm{~min}$, after which the samples were electrophoresed in $10 \%$ SDS-polyacrylamide gel and transferred to a 4.5- $\mu \mathrm{m}$ nitrocellulose membrane. After blocking in $5 \%$ nonfat milk for $45 \mathrm{~min}$ at room temperature (RT), the membranes were incubated at $4{ }^{\circ} \mathrm{C}$ overnight with the following primary antibodies raised in rabbit against: L12-LOX (1:1,000), 5-LOX (1:600) (Cayman Chemical, Ann Arbor, USA), ChemR23 (1:500), and ALX/FPR2 (1:500) (Santa Cruz, CA, USA). After washing, the membranes were incubated with horseradish peroxidase (HRP)-conjugated secondary antibodies for $2 \mathrm{~h}$ at RT. Finally, the membranes were developed by ECL reagent (GE health Care, Buckinghamshire, UK), and the signals detected by a CCD camera (Fuji Film, Tokyo, Japan). Analysis of the bands was carried out using the MultiGauge software (Fuji Film, Tokyo, Japan).

\section{Immunohistochemistry}

The fixed brains ( $n=4$ per group except $n=5$ for the 9-monthold SAMP8 group) were sectioned at $12 \mu \mathrm{m}$ thickness in a cryostat (Leica Microsystems, IL, USA) and mounted onto polarized glass slides. The sections were kept at $-20^{\circ} \mathrm{C}$ until further analysis. For immunofluorescent staining, the sections were blocked for $30 \mathrm{~min}$ at RT with $5 \%$ normal serum and then incubated overnight at $4{ }^{\circ} \mathrm{C}$ with primary antibodies as follows: rat anti-F4/80 1:100 (AbD Serotec, Puchheim, Germany); rabbit anti-ALX/FPR2 1:100 (Santa Cruz, CA, USA); and rabbit anti-ChemR23 1:200, anti-L12-LOX 1:200, and anti-5-LOX 1:200 (all from Cayman Chemical, Ann Arbor, USA). After washing, the slides were incubated with the appropriate secondary antibodies for $1 \mathrm{~h}$ at RT, washed, and then mounted in fluorescence mounting medium (Dako, Stockholm, Sweden).

Double labeling was performed on sections incubated with a mixture of (i) one of the above primary antibodies, and the neuronal marker, mouse anti-NeuN 1:500 (Chemicon/
Millipore, Billerica, MA, USA); (ii) primary antibodies to 5LOX and antibodies for the astrocyte marker rat anti-glial fibrillary acidic protein (GFAP, 1:400; Invitrogen, Carlsbad, CA, USA), or the microglial marker rat anti-F4/80 (1:100; AbD Serotec, Puchheim, Germany); (iii) rabbit anti-L12-LOX 1:200 and mouse anti-A $\beta$ 4G8 1:100 (Signet/Covance, Dedham, MA, USA). A mixture of the appropriate secondary antibodies was used to detect the staining. Analysis was carried out under a fluorescence microscope (Nikon Eclipse 800, Tokyo, Japan).

\section{Statistical Analysis}

Statistical analysis was performed with SPSS software (version 21.0, IBM Corporation, NY, USA). The univariate general linear model (GLM) was used, considering strain and age as two dependent variables. $p$ values less than 0.05 were considered as statistically significant. All statistical data are expressed as mean \pm standard error of the mean (SEM).

\section{Results}

Levels of Pro-resolving and Pro-inflammatory Markers

Pro-resolving and pro-inflammatory profiles were analyzed in the hippocampus of SAMP8 and SAMR1 mice. Levels of $\mathrm{LXA}_{4}$ and RvD1 were not different between SAMP8 and agematched SAMR1 mice, nor between 2- and 9-month-old mice in either strain (Fig. 1a, b).

The pro-inflammatory profile was assessed by analysis of chemerin, MHC-II, and F4/80. Levels of chemerin, a chemoattractant protein with proinflammatory effects, were higher in 9- than in 2month-old SAMP8 mice. In the SAMR1 strain, there was a trend, albeit not statistically significant, toward lower levels of chemerin at 9 months (Fig. 1c). Higher levels of MHC-II were found in the hippocampus of both 2- and 9-month-old SAMP8 mice as compared to age-matched SAMR1 (Fig. 1d). In addition, the MHC-II levels were higher in 9- than in 2-month-old SAMP8 mice (Fig. 1d). Analysis of F4/80, a marker for activated microglia, showed similar staining in SAMP8 and SAMR 1 mice at 2 months of age, whereas at 9 months of age, a higher staining intensity was observed in SAMP8 mice as compared to age-matched SAMR1 mice (Fig. 1e).

\section{SPM Receptors}

In view of unchanged $\mathrm{LXA}_{4}$ and RvD1 levels during aging in SAMP8 mice, we next investigated if there was a change in 
A
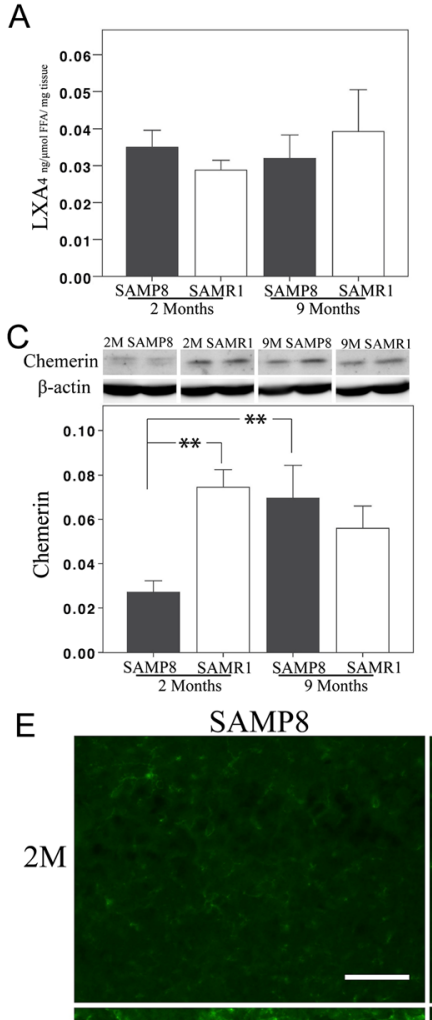

SAMP8
B

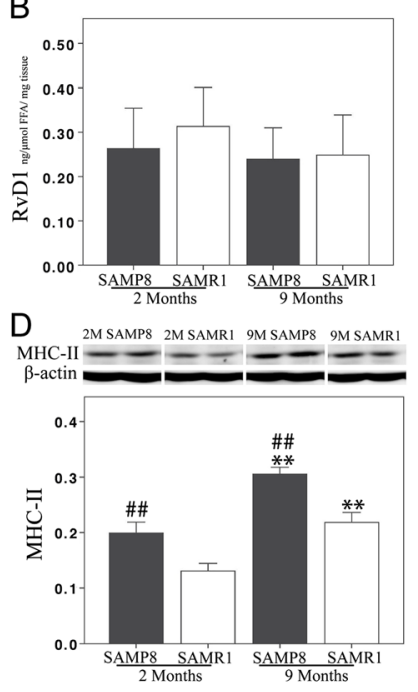
SAMR1

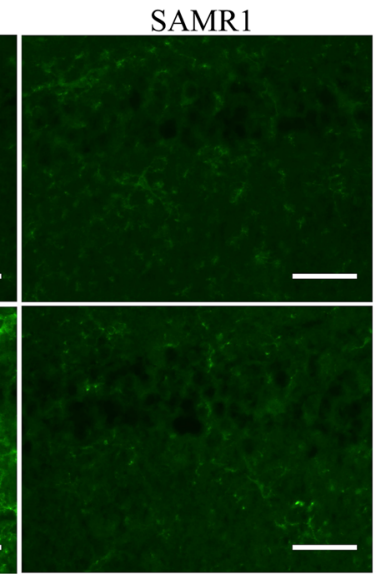

Fig. 1 a-e SPMs and pro-inflammatory markers in the hippocampus of SAMP8 and SAMR1 mice. a, b Unchanged levels of $\mathrm{LXA}_{4}$ and RvD1 in SAMP8 mice compared to age-matched controls, and in both strains upon aging, as assessed by EIA. c Western blot data show lower levels of chemerin in SAMP8 mice at 2 months of age, but an increase with age reaching the same level as in SAMR1 mice at 9 months; ${ }^{* *} p<0.01$. $\mathbf{d}$ The MHC-II levels are higher in SAMP8 mice at both 2 and 9 months of age (\#\#p<0.01, SAMP8 vs. age-matched SAMR1), and the SAMR1 mice show a decrease with age $\left({ }^{*} p<0.01,9\right.$ vs. 2 months). e A markedly stronger microglial staining for F4/80 is seen in SAMP8 mice compared to SAMR1 mice at 9 months, as well as an increase with age in SAMP8 mice. Scale bar $=40 \mu \mathrm{m}$. Data are presented as mean \pm SEM

their receptors. Immunohistochemical analysis showed staining of ALX/FPR2 in pyramidal cells in the hippocampus of both SAMP8 and SAMR1 mice (Fig. 2a, c). The neuronal staining was considerably weaker in cornu ammonis area 1 (CA1) than in CA2-4 (Fig. 2c). There was an increase with age in both SAMP8 and SAMR1 mice, but no difference between the two mouse strains, as shown both by immunohistochemistry and western blot (Fig. 2a, b).

Analysis of ChemR23, the receptor for RvE1 and chemerin, showed a similar distribution as that for ALX/ FPR2 (Fig. 2d, f), i.e., staining of pyramidal cells, and with higher intensity in CA2-4. At 9 months of age, the

hippocampal levels of ChemR23 were lower in SAMP8 than in SAMR1 mice, whereas no difference could be seen between the young and older mice in either strain (Fig. 2e).

Enzymes Involved in SPM Biosynthesis

L12-LOX is the first enzyme to catalyze arachidonic acid (AA) or docosahexaenoic acid (DHA) during the production of $\mathrm{LXA}_{4}$ (Serhan et al. 1986) or RvD1 (Sun et al. 2007). It is functionally and structurally similar to 15 -LOX-1 and sometimes named as 12/15-LOX together with 15-LOX-1 (L12LOX is used through out the current study) (Haeggstrom and Funk 2011). Immunohistochemical analysis showed staining of L12-LOX in pyramidal neurons (Fig. 3a, c) throughout CA1 to CA4. Interestingly, clusters of punctate immunofluorescence for L12-LOX were observed in the hippocampus of 9-month-old SAMP8 mice, but not in anyone of the other animal groups (Figs. 3a and 5a). Western blot analysis demonstrated lower levels of L12-LOX in SAMP8 compared to SAMR1 mice at 9 months (Fig. 3b). Aging did not affect the L12-LOX levels in SAMP8 mice, but an increase with age was seen in the SAMR1 strain.

5-LOX is the second enzyme involved in the production of $\mathrm{LXA}_{4}$ (Serhan et al. 1986) and RvD1 (Sun et al. 2007). In the hippocampus of both SAMP8 and SAMR1 mice, 5-LOX immunostaining was found in neurons and glial cells (Fig. 4a). As shown by double immunohistochemistry with GFAP, 5-LOX staining can be seen in both pyramidal neurons and astrocytes (Fig. 4c). There was no co-localization with the microglial marker F4/80 (Fig. 4c). The overall change in 5LOX levels was assessed by western blot. The levels were lower in 2-month-old and higher at 9-month-old SAMP8 compared to age-matched SAMR1 mice (Fig. 4b). SAMP8 mice showed no change in 5-LOX levels with age. In contrast, a significant decrease in 5-LOX was found in 9-month-old SAMR1 compared with 2-month-old SAMR1 mice (Fig. 4b).

\section{AD-Related Markers}

Analysis of L12-LOX revealed clustered immunostaining in the group of 9-month-old SAMP8 mice, but not in any other group (Fig. 3a). The staining pattern is similar to that described previously for $\mathrm{A} \beta$ deposits in aging SAMP8 mice (Del Valle et al. 2010; Manich et al. 2011). Double immunostaining with 4G8 antibodies (also used in Del Valle et al. 2010; Manich et al. 2011) revealed partial co-localization of L12-LOX and $A \beta$ in the hippocampus of 9-month-old SAMP8 mice (Fig. 5a).

The levels of P-tau were analyzed by western blot using AT-8 antibodies (Ser202/Thr205 p-tau). Hippocampal P-tau levels were significantly higher in 9-month-old SAMP8 compared to SAMR1 mice of the same age (Fig. 5b). There was an increase with age in both strains (Fig. 5b). Correlation analysis 
Fig. 2 a-f Analysis of ALX/ FPR2, the receptor for $\mathrm{LXA}_{4}$ and RvD1, and ChemR23, the receptor for RvE1, in SAMP8 and SAMR1 mice. a Immunofluorescence staining for ALX/FPR2 in pyramidal cells in the CA2-3 regions. $\mathbf{b}$ Data from western blot analysis of the hippocampus show higher levels of ALX/FPR2 at 9 months of age in both strains, but no difference between the SAMP8 and SAMR1 mice. c Double staining shows localization of ALX/FPR2 in NeuN-positive pyramidal neurons in the CA2 region. The ALX/ FPR2 labeling is markedly weaker in CA1 than in CA2. d Immunofluorescence staining of ChemR23 in pyramidal cells in the CA2-3 regions. e Data from western blot analysis of the hippocampus show lower levels of ChemR23 in SAMP8 mice compared to SAMR1 at 9 months. There is no difference with age in either strain. f Double staining shows localization of ChemR23 in NeuN-positive pyramidal neurons in the CA2 region. No staining for ChemR23 can be seen in CA1. Scale bar= $40 \mu \mathrm{m}$. Data are presented as mean \pm SEM. $* p<0.05, * * p<0.01$

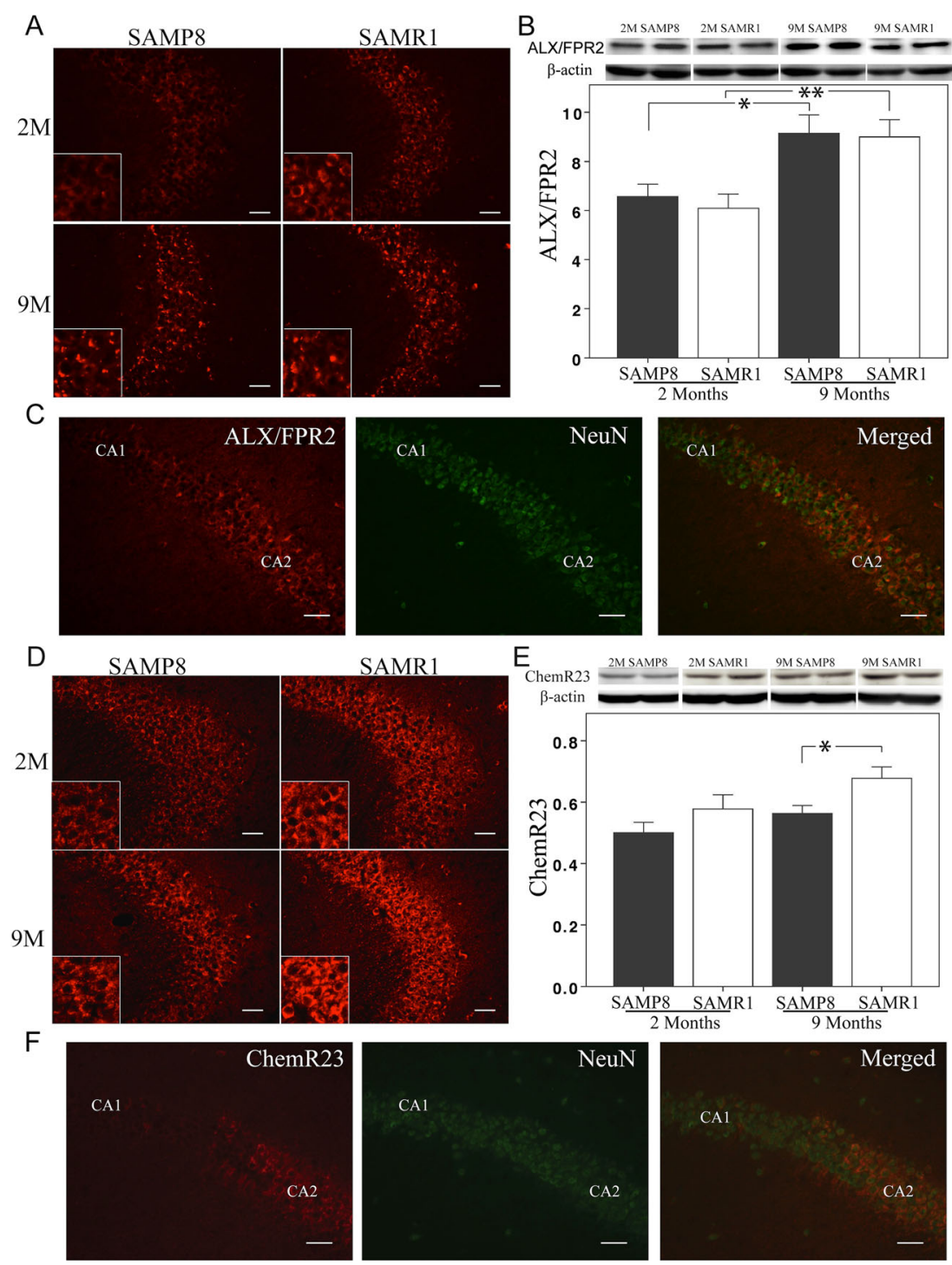

between AT-8 and L12-LOX showed a positive correlation (Pearson correlation test, $r=0.679, p<0.05$ ) in the SAMP8 strain (Fig. 5c), but not in the SAMR1 strain.

\section{Discussion}

Resolution of inflammation mediated by SPMs emerges as an important factor in various inflammation-associated diseases, including $\mathrm{AD}$. In previous studies, we have demonstrated lower levels of the pro-resolving factor $\mathrm{LXA}_{4}$ in cerebrospinal fluid (CSF) samples from AD patients, as well as higher levels of SPM receptors and a biosynthetic enzyme, in the AD hippocampus (Wang et al. 2014). In the present study, by using SAMP8 and SAMR1 mice, we investigated how the resolution pathway is regulated in aging, the primary risk factor for $\mathrm{AD}$.
Healthy aging is associated with low-grade inflammation and is sometimes referred to as "inflamm-aging" (Franceschi et al. 2000). In a previous study, IL-1 $\beta$ levels were elevated with age in the SAMR1 strain (Tha et al. 2000). In agreement with this, we found a slight increase associated with aging in the microglial activation marker MHC-II in SAMR1 mice. This was accompanied by unchanged levels of the SPMs $\mathrm{LXA}_{4}$ and RvD1, whereas ALX/FPR2, the receptor for $\mathrm{LXA}_{4}$ and RvD1, was upregulated with age. These data demonstrate the regulation of pro-resolving signaling during a healthy aging progress (see schematic Fig. 6). Moreover, since L12-LOX and 5-LOX are both needed to sequentially oxidize AA or DHA to produce $\mathrm{LXA}_{4}$ or RvD1, it is hypothesized that the stable levels of $\mathrm{LXA}_{4}$ and RvD1 with age in SAMR1 mice may be a consequence of increased L12-LOX levels, but decreased levels of 5-LOX.

The SAMP8 strain is a model of accelerated aging, obtained by selecting for this phenotype from the same background 
Fig. 3 a-c Analysis of L12LOX. a Immunofluorescence staining of L12-LOX in pyramidal cells. In 9-month-old SAMP8 mice, clusters of punctate staining can be seen in the hippocampus (arrows). b Data from western blot analysis of the hippocampus show lower levels of L12-LOX in SAMP8 mice compared to SAMR1 mice at 9 months. There is no difference with age in the SAMP8 strain, but an increase in the SAMR1 strain. c Double staining shows localization of L12-LOX in NeuN-positive pyramidal neurons. Scale bar $=40 \mu \mathrm{m}$. Data are presented as mean \pm SEM. $* * p<0.01$

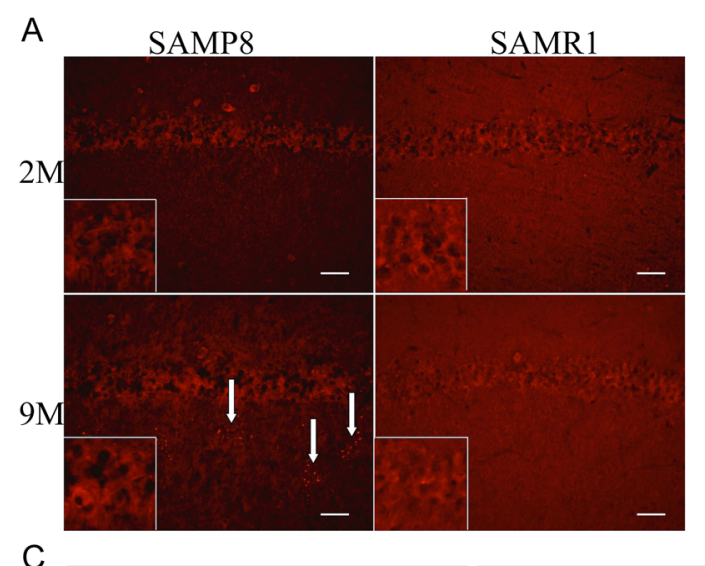

B
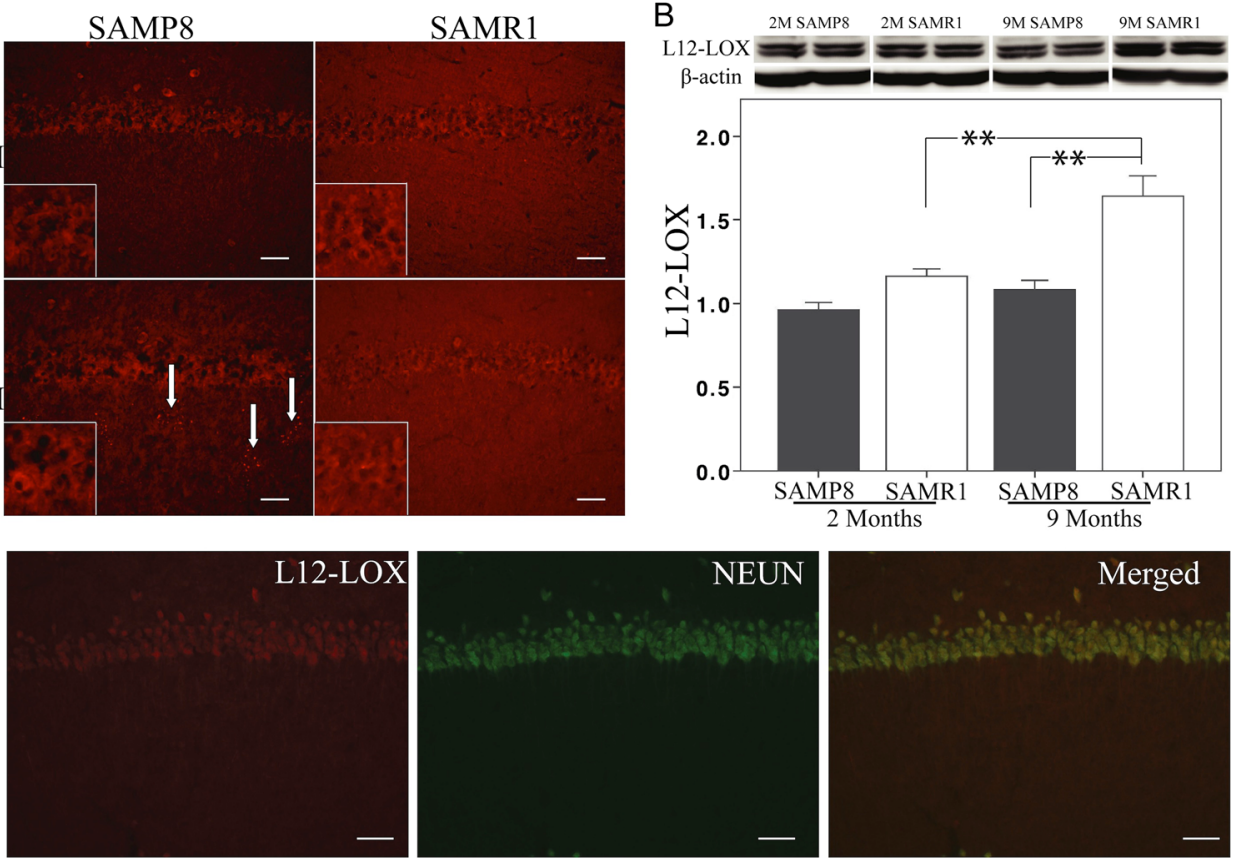

Fig. 4 a-c Analysis of 5-LOX. a 5 -LOX is seen in both pyramidal neurons and glia. b Data from western blot analysis of the hippocampus show lower levels of 5-LOX in 2-month-old SAMP8 mice and higher levels in 9-month-old SAMP8 mice, compared to age-matched SAMR1 mice. There is no difference with age in the SAMP8 strain, but a decrease in the SAMR1 strain. c Double staining shows localization of 5-LOX in NeuN-positive pyramidal neurons (arrows and arrowhead in top panel indicate cell with colocalization), as well as in GFAP-positive astrocytes (arrows and arrowhead in middle panel indicate cell with colocalization), but not in F4/80-positive microglia (arrows and arrowhead in lower panel indicate separate cells). Scale bar $=40 \mu \mathrm{m}$. Data are presented as mean $\pm \mathrm{SEM}$. ${ }^{*} p<0.05, * * p<0.01$
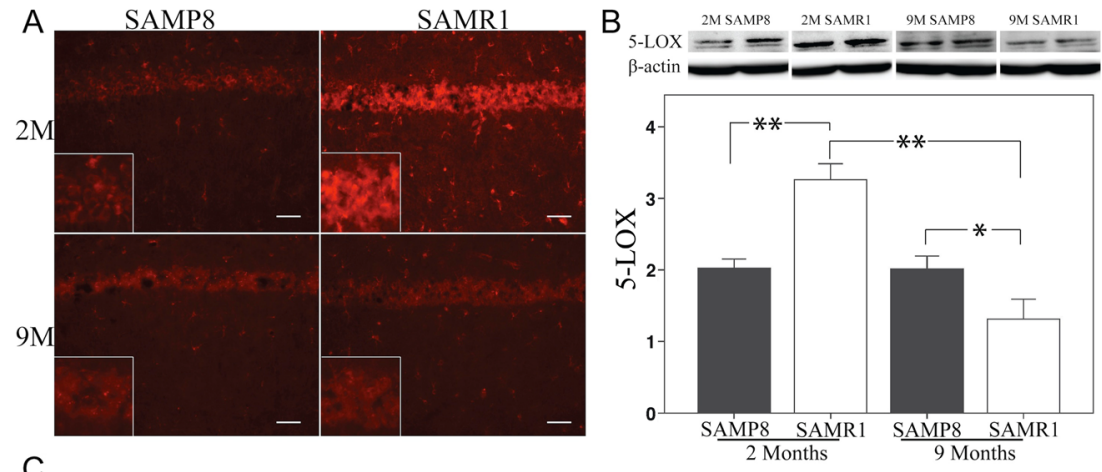

C
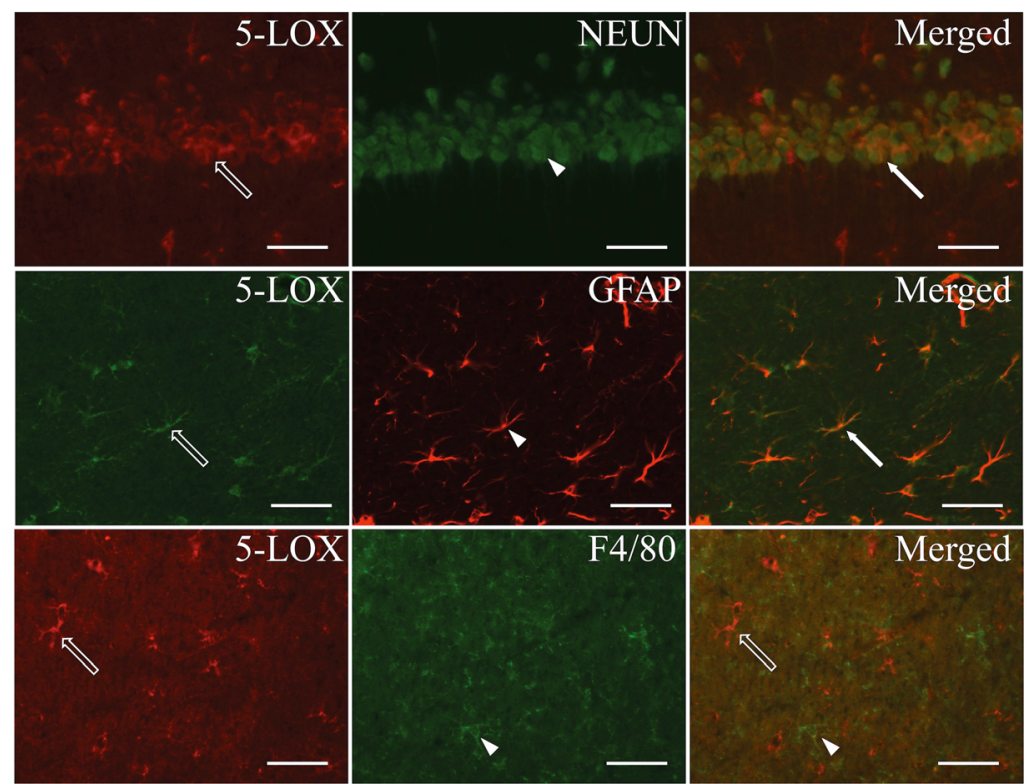
Fig. 5 a-c Analysis of L12-LOX in relation to $A \beta$ and $P$-tau in SAMP8 and SAMR1 mice. a Double staining shows the colocalization (arrows) of L12LOX with A $\beta$ (arrowheads) (using the 4G8 antibody) in clusters of punctate structures in the hippocampus of 9-month-old SAMP8 mice. b Levels of AT-8 P-tau are higher in 9-month-old SAMP8 than in age-matched SAMR1 mice. Data from western blot analysis of the hippocampus show an increase with age in Ptau levels in both strains. Bar= $40 \mu \mathrm{m}$. c Positive correlation between the levels of L12-LOX and AT-8 P-tau (Pearson correlation test, $r=0.679$, $p<0.05)$. Data are presented as mean \pm SEM. \#\# $p<0.01$, SAMP8 vs. age-matched SAMR1; $*^{* *} p<0.01,9$ vs. 2 months

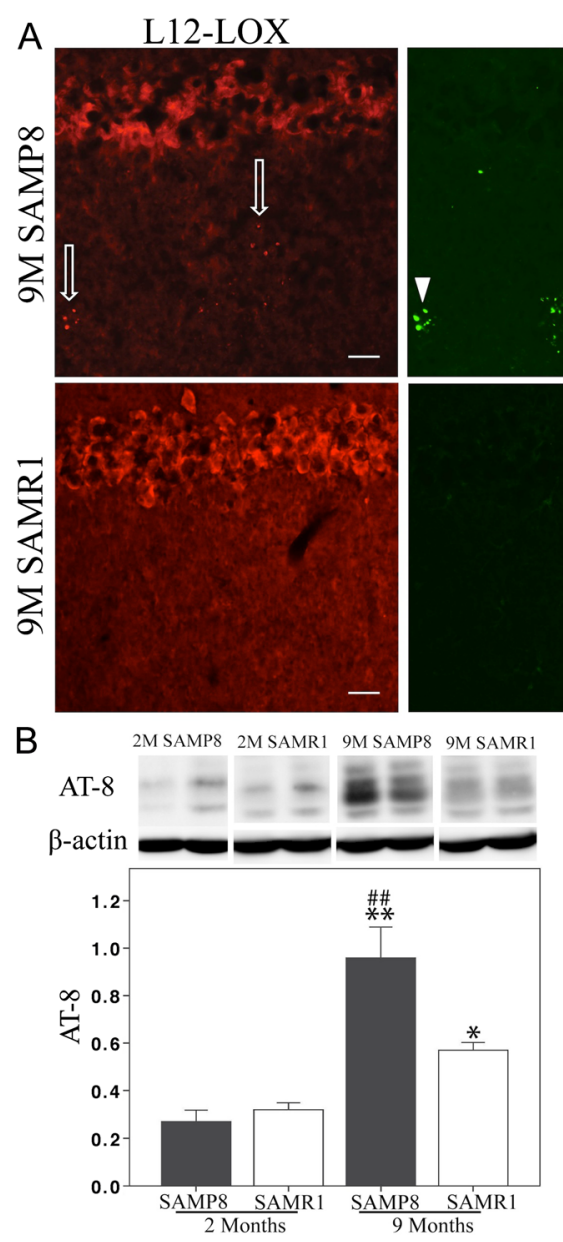

$4 \mathrm{G} 8$

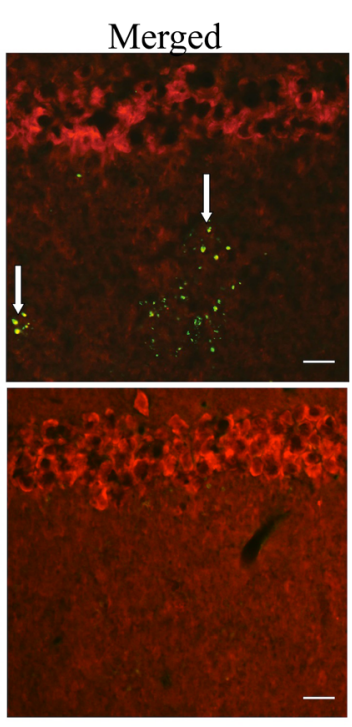

C

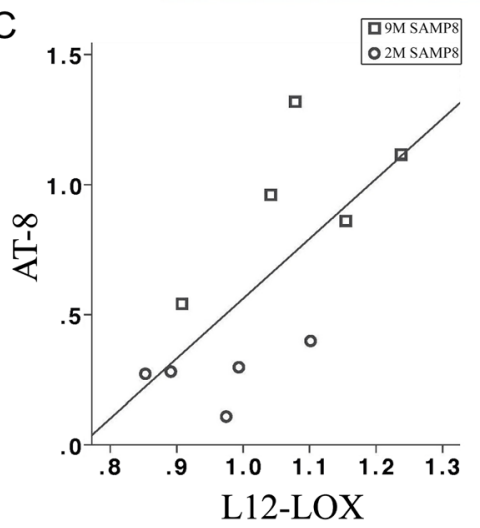

strain as SAMR1 (Takeda et al. 1981; Miyamoto et al. 1986). Thus, without being transgenic for human AD mutations, SAMP8 mice display impaired learning and memory and increased $A \beta$ levels and phosphorylation of tau with age (Pallas et al. 2008). In the present study, SAMP8 mice showed a more marked increase in inflammation upon aging as compared to normal aging SAMR1 mice. This was shown by higher levels of MHC-II and F4/80 in aged SAMP8 mice, confirming previous studies showing increased levels of the pro-inflammatory cytokines IL- $1 \beta$, IL-6, and TNF- $\alpha$ (Tha et al. 2000; Liu et al. 2013). Furthermore, the levels of chemerin were dramatically increased upon age in SAMP8 mice, whereas no significant change was seen in SAMR1 mice. Chemerin has been proposed as a pro-inflammatory marker in multiple sclerosis, due to its function as a chemoattractant for inflammatory cells (Lande et al. 2008; Graham et al. 2009). There was no significant change in the levels of its receptor ChemR23, but the resulting elevated chemerin/ChemR23 axis in abnormal aging lends further evidence for a state of exaggerated inflammation.

The resolution response regulates inflammation by an active process mediated by SPMs (Recchiuti and Serhan 2012), and the excessive inflammation in the aged SAMP8 mice may require a stronger pro-resolving signaling by SPMs compared to the baseline in SAMR1 mice. However, there was no difference in the levels of $\mathrm{LXA}_{4}$ and RvD1 between the aged SAMP8 and the aged SAMR1 mice, even though the inflammation was more pronounced in SAMP8 mice. The levels of the receptor ALX/FPR2 were elevated upon aging in SAMP8 mice, but were not different from the levels in age-matched SAMR1 mice. This indicates that the resolution axis, i.e., $\mathrm{LXA}_{4} / \mathrm{RvD} 1-\mathrm{ALX} / \mathrm{FPR} 2$, remained at a baseline level similar to SAMR1 mice. Thus, the resolution in SAMP8 mice could be considered unresponsive to the inflammation. It may be hypothesized that the cause of this unresponsiveness is the increased AD-like molecular pathology present in SAMP8 mice. Notably, A $\beta$ levels increase with age in SAMP8 mice (Takemura et al. 1993; Del Valle et al. 2010; Manich et al. 2011; and our own data), and this neurotoxic peptide also binds to ALX/FPR2 (Le et al. 2001; Yazawa et al. 2001). As a result, the net effect of competition between increased $A \beta$ and unchanged $\mathrm{LXA}_{4}$ and RvD1 may be decreased pro-resolving signaling and increased harmful signal transduction through ALX/FPR2 (see schematic Fig. 6b).

The LOX enzymes govern the biosynthesis of SPMs, but they also have other distinctive functions, complicating the 


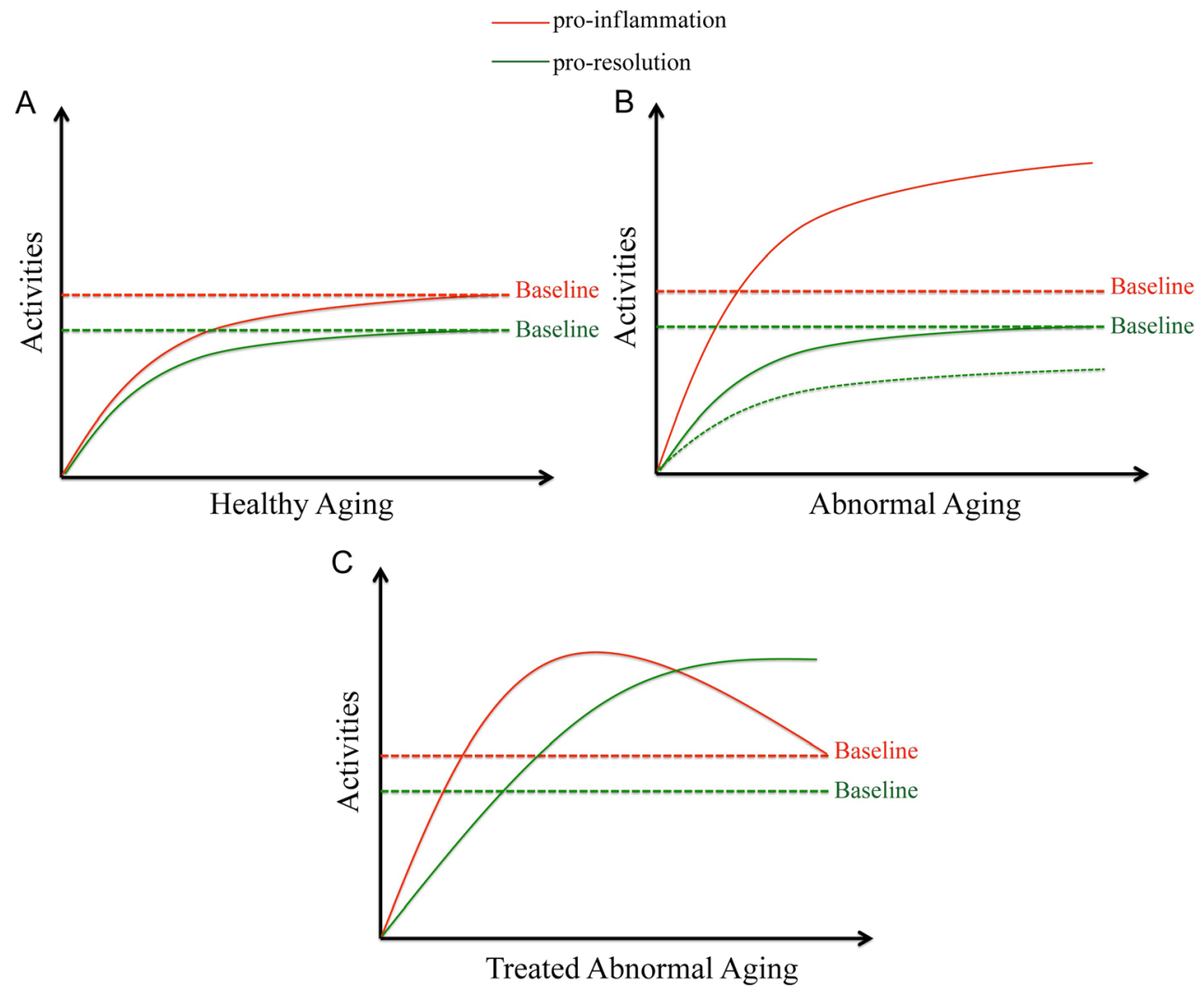

Fig. 6 a-c Schematic representations of the hypothesis for resolution in healthy aging, abnormal aging related to $\mathrm{AD}$, and treatment for abnormal aging, as concluded from the data obtained in SAMP8 and SAMR1 mice. a Healthy aging (baselines for inflammation and resolution) is characterized by a small increase in pro-inflammatory activities, accompanied by increased pro-resolving activities (due to increased SPM receptor levels). b Abnormal aging related to $\mathrm{AD}$ develops excessive pro-inflammatory

interpretation of their role. For example, elevated levels of L12-LOX in response to skin inflammation were associated with enhanced $\mathrm{LXA}_{4}$ biosynthesis (Levy et al. 2001), and overexpression of L12-LOX was beneficial to a rabbit periodontitis model due to more production of $\mathrm{LXA}_{4}$ (Serhan et al. 2003). However, overexpression of L12-LOX has also been shown to increase $A \beta$ production (Chu et al. 2012) and enhanced tau-phosphorylation at Ser202/Thr205 (AT-8) (Giannopoulos et al. 2013) in Tg2576 APP transgenic mice. Here we show co-localization of L12-LOX with the A $\beta$ clusters found in aged SAMP8 mice (Del Valle et al. 2010; Manich et al. 2011), indicating a potential novel association between L12-LOX and A $\beta$. We also found a positive correlation between L12-LOX and tau-phosphorylation at Ser202/ Thr205 in SAMP8, but not in SAMR1 mice. These data may indicate a detrimental role of L12-LOX in aged SAMP8 mice. However, there were higher L12-LOX levels also in the aged SAMR1 mice, but without correlation with AD-like pathology. The paradox of L12-LOX function found in the present study indicates the need for further studies to characterize the activities as compared to baseline (i.e., upon aging in SAMR1 mice), but the pro-resolving activities are insufficient and remain at baseline levels that meet only the baseline inflammation. The dashed curve indicates that pro-resolving activities may be even lower than baseline, due to increased levels of $A \beta$, binding to ALX/FPR2. $\mathbf{c}$ Treatment including supplement of SPMs or modulation of LOXs may resolve the excessive inflammation in abnormal aging

regulation of L12-LOX function, e.g., regarding differential phosphorylation (Khanna et al. 2005). Notably, in addition to increased L12-LOX, we found that 5-LOX was decreased with age in SAMR1 mice. The same counter-regulation of L12-LOX and 5-LOX has been reported in IL-4-treated dendritic cells and was proposed as a mechanism mediating immune modulation and anti-inflammatory effects (Spanbroek et al. 2001). In the abnormal aging SAMP8 mice, this counter-regulation did not happen, and the two LOXs remained at unchanged levels with age. This absence of counter-regulation of L12-LOX and 5-LOX may contribute to dysfunction of resolution in SAMP8 mice.

In summary, we provided data on how the resolution pathway in the brain is regulated to meet inflammation during normal aging using an animal model. Moreover, during abnormal aging, accompanied by AD-like pathologies, there was insufficient resolution response to resolve the excessive inflammation compared to normal aging. This insufficiency may be partially due to dysregulation of LOXs, and their role during development of $\mathrm{AD}$-like pathologies needs further 
characterization. Future studies will be required to establish whether supplementary treatment with SPMs or modulating LOXs may ameliorate aging-related $\mathrm{AD}$ pathologies by reestablishing the balance between resolution and inflammation (see schematic Fig. 6c).

Open Access This article is distributed under the terms of the Creative Commons Attribution License which permits any use, distribution, and reproduction in any medium, provided the original author(s) and the source are credited.

\section{References}

Arita M, Bianchini F, Aliberti J, Sher A, Chiang N, Hong S, Yang R, Petasis NA, Serhan CN (2005) Stereochemical assignment, antiinflammatory properties, and receptor for the omega-3 lipid mediator resolvin E1. J Exp Med 201:713-722

Bannenberg G, Serhan CN (2010) Specialized pro-resolving lipid mediators in the inflammatory response: an update. Biochim Biophys Acta 1801:1260-1273

Campuzano O, Castillo-Ruiz MM, Acarin L, Castellano B, Gonzalez B (2009) Increased levels of proinflammatory cytokines in the aged rat brain attenuate injury-induced cytokine response after excitotoxic damage. J Neurosci Res 87:2484-2497

Carpenter AF, Carpenter PW, Markesbery WR (1993) Morphometric analysis of microglia in Alzheimer's disease. J Neuropathol Exp Neurol 52:601-608

Chu J, Zhuo JM, Pratico D (2012) Transcriptional regulation of $\beta$ secretase-1 by $12 / 15$-lipoxygenase results in enhanced amyloidogenesis and cognitive impairments. Ann Neurol 71:57-67

de Gonzalo-Calvo D, Neitzert K, Fernandez M, Vega-Naredo I, Caballero B, Garcia-Macia M, Suarez FM, Rodriguez-Colunga MJ, Solano JJ, Coto-Montes A (2010) Differential inflammatory responses in aging and disease: TNF- $\alpha$ and IL- 6 as possible biomarkers. Free Radic Biol Med 49:733-737

Del Valle J, Duran-Vilaregut J, Manich G, Casadesus G, Smith MA, Camins A, Pallas M, Pelegri C, Vilaplana J (2010) Early amyloid accumulation in the hippocampus of SAMP8 mice. J Alzheimers Dis 19:1303-1315

Fiore S, Maddox JF, Perez HD, Serhan CN (1994) Identification of a human cDNA encoding a functional high affinity lipoxin A4 receptor. J Exp Med 180:253-260

Franceschi C, Bonafe M, Valensin S, Olivieri F, De Luca M, Ottaviani E, De Benedictis G (2000) Inflamm-aging. An evolutionary perspective on immunosenescence. Ann N Y Acad Sci 908:244-254

Giannopoulos PF, Joshi YB, Chu J, Pratico D (2013) The 12-15lipoxygenase is a modulator of Alzheimer's-related tau pathology in vivo. Aging Cell 12:1082-1090

Graham KL, Zabel BA, Loghavi S, Zuniga LA, Ho PP, Sobel RA, Butcher EC (2009) Chemokine-like receptor-1 expression by central nervous system-infiltrating leukocytes and involvement in a model of autoimmune demyelinating disease. J Immunol 183:6717-6723

Haeggstrom JZ, Funk CD (2011) Lipoxygenase and leukotriene pathways: biochemistry, biology, and roles in disease. Chem Rev 111: 5866-5898

Herrup K (2010) Reimagining Alzheimer's disease - an age-based hypothesis. J Neurosci 30:16755-16762

in't Veld BA, Ruitenberg A, Hofman A, Launer LJ, van Duijn CM, Stijnen T, Breteler MM, Stricker BH (2001) Nonsteroidal antiinflammatory drugs and the risk of Alzheimer's disease. N Engl J Med 345:1515-1521
Kawas C, Gray S, Brookmeyer R, Fozard J, Zonderman A (2000) Agespecific incidence rates of Alzheimer's disease: the Baltimore Longitudinal Study of Aging. Neurology 54:2072-2077

Khanna S, Roy S, Slivka A, Craft TK, Chaki S, Rink C, Notestine MA, DeVries AC, Parinandi NL, Sen CK (2005) Neuroprotective properties of the natural vitamin E alpha-tocotrienol. Stroke 36:22582264

Krishnamoorthy S, Recchiuti A, Chiang N, Fredman G, Serhan CN (2012) Resolvin D1 receptor stereoselectivity and regulation of inflammation and proresolving microRNAs. Am J Pathol 180: 2018-2027

Lande R, Gafa V, Serafini B, Giacomini E, Visconti A, Remoli ME, Severa M, Parmentier M, Ristori G, Salvetti M, Aloisi F, Coccia EM (2008) Plasmacytoid dendritic cells in multiple sclerosis: intracerebral recruitment and impaired maturation in response to interferon$\beta$. J Neuropathol Exp Neurol 67:388-401

Le Y, Gong W, Tiffany HL, Tumanov A, Nedospasov S, Shen W, Dunlop NM, Gao JL, Murphy PM, Oppenheim JJ, Wang JM (2001) Amyloid (beta)42 activates a G-protein-coupled chemoattractant receptor, FPR-like-1. J Neurosci 21:RC123

Levy BD, Clish CB, Schmidt B, Gronert K, Serhan CN (2001) Lipid mediator class switching during acute inflammation: signals in resolution. Nat Immunol 2:612-619

Liu J, Hu G, Xu R, Qiao Y, Wu HP, Ding X, Duan P, Tu P, Lin YJ (2013) Rhein lysinate decreases the generation of $\beta$-amyloid in the brain tissues of Alzheimer's disease model mice by inhibiting inflammatory response and oxidative stress. J Asian Nat Prod Res 15:756763

Manich G, Mercader C, del Valle J, Duran-Vilaregut J, Camins A, Pallas M, Vilaplana J, Pelegri C (2011) Characterization of amyloid- $\beta$ granules in the hippocampus of SAMP8 mice. J Alzheimers Dis 25:535-546

McGeer PL, Itagaki S, Tago H, McGeer EG (1987) Reactive microglia in patients with senile dementia of the Alzheimer type are positive for the histocompatibility glycoprotein HLA-DR. Neurosci Lett 79: 195-200

McGeer P, McGeer E, Rogers J, Sibley J (1990) Anti-inflammatory drugs and Alzheimer disease. Lancet 335:1037

Miyamoto M, Kiyota Y, Yamazaki N, Nagaoka A, Matsuo T, Nagawa Y, Takeda T (1986) Age-related changes in learning and memory in the senescence-accelerated mouse (SAM). Physiol Behav 38:399-406

Ogura K, Ogawa M, Yoshida M (1994) Effects of ageing on microglia in the normal rat brain: immunohistochemical observations. Neuroreport 5:1224-1226

Pallas M, Camins A, Smith MA, Perry G, Lee HG, Casadesus G (2008) From aging to Alzheimer's disease: unveiling "the switch" with the senescence-accelerated mouse model (SAMP8). J Alzheimers Dis $15: 615-624$

Recchiuti A, Serhan CN (2012) Pro-resolving lipid mediators (SPMs) and their actions in regulating miRNA in novel resolution circuits in inflammation. Front Immunol 3:298

Sastre M, Walter J, Gentleman SM (2008) Interactions between APP secretases and inflammatory mediators. J Neuroinflammation 5:25

Serhan CN (2007) Resolution phase of inflammation: novel endogenous anti-inflammatory and proresolving lipid mediators and pathways. Annu Rev Immunol 25:101-137

Serhan CN (2010) Novel lipid mediators and resolution mechanisms in acute inflammation: to resolve or not? Am J Pathol 177:1576-1591

Serhan CN (2011) The resolution of inflammation: the devil in the flask and in the details. FASEB J 25:1441-1448

Serhan CN, Nicolaou KC, Webber SE, Veale CA, Dahlen SE, Puustinen TJ, Samuelsson B (1986) Lipoxin A. Stereochemistry and biosynthesis. J Biol Chem 261:16340-16345

Serhan CN, Jain A, Marleau S, Clish C, Kantarci A, Behbehani B, Colgan SP, Stahl GL, Merched A, Petasis NA, Chan L, Van Dyke TE (2003) Reduced inflammation and tissue damage in transgenic rabbits 
overexpressing 15-lipoxygenase and endogenous anti-inflammatory lipid mediators. J Immunol 171:6856-6865

Serhan CN, Krishnamoorthy S, Recchiuti A, Chiang N (2011) Novel antiinflammatory-pro-resolving mediators and their receptors. Curr Top Med Chem 11:629-647

Serrano-Pozo A, Mielke ML, Gomez-Isla T, Betensky RA, Growdon JH, Frosch MP, Hyman BT (2011) Reactive glia not only associates with plaques but also parallels tangles in Alzheimer's disease. Am J Pathol 179:1373-1384

Sheffield LG, Berman NE (1998) Microglial expression of MHC class II increases in normal aging of nonhuman primates. Neurobiol Aging 19:47-55

Spanbroek R, Hildner M, Kohler A, Muller A, Zintl F, Kuhn H, Radmark O, Samuelsson B, Habenicht AJ (2001) IL-4 determines eicosanoid formation in dendritic cells by down-regulation of 5-lipoxygenase and up-regulation of 15-lipoxygenase 1 expression. Proc Natl Acad Sci U S A 98:5152-5157

Stewart WF, Kawas C, Corrada M, Metter EJ (1997) Risk of Alzheimer's disease and duration of NSAID use. Neurology 48:626-632

Sun YP, Oh SF, Uddin J, Yang R, Gotlinger K, Campbell E, Colgan SP, Petasis NA, Serhan CN (2007) Resolvin D1 and its aspirin-triggered $17 \mathrm{R}$ epimer. Stereochemical assignments, anti-inflammatory properties, and enzymatic inactivation. J Biol Chem 282:9323-9334

Swardfager W, Lanctot K, Rothenburg L, Wong A, Cappell J, Herrmann N (2010) A meta-analysis of cytokines in Alzheimer's disease. Biol Psychiatry 68:930-941

Takeda T, Hosokawa M, Takeshita S, Irino M, Higuchi K, Matsushita T, Tomita Y, Yasuhira K, Hamamoto H, Shimizu K, Ishii M, Yamamuro T (1981) A new murine model of accelerated senescence. Mech Ageing Dev 17:183-194
Takemura M, Nakamura S, Akiguchi I, Ueno M, Oka N, Ishikawa S, Shimada A, Kimura J, Takeda T (1993) Beta/A4 proteinlike immunoreactive granular structures in the brain of senescence-accelerated mouse. Am J Pathol 142:1887-1897

Tha KK, Okuma Y, Miyazaki H, Murayama T, Uehara T, Hatakeyama R, Hayashi Y, Nomura Y (2000) Changes in expressions of proinflammatory cytokines IL- $1 \beta$, TNF- $\alpha$ and IL- 6 in the brain of senescence accelerated mouse (SAM) P8. Brain Res 885:25-31

Vom BJ, Prokop S, Miller KR, Obst J, Kalin RE, Lopategui-Cabezas I, Wegner A, Mair F, Schipke CG, Peters O, Winter Y, Becher B, Heppner FL (2012) Inhibition of IL-12/IL-23 signaling reduces Alzheimer's disease-like pathology and cognitive decline. Nat Med 18:1812-1819

Wang X, Zhu M, Hjorth E, Cortes-Toro V, Eyjolfsdottir H, Graff C, Nennesmo I, Palmblad J, Eriksdotter M, Sambamurti K, Fitzgerald JM, Serhan CN, Granholm AC, Schultzberg M (2014) Resolution of inflammation is altered in Alzheimer's disease. Alzheimers Dement. doi:10.1016/j.jalz.2013.12.024

Wei J, Xu H, Davies JL, Hemmings GP (1992) Increase of plasma IL-6 concentration with age in healthy subjects. Life Sci 51:1953-1956

Wittamer V, Franssen JD, Vulcano M, Mirjolet JF, Le Poul E, Migeotte I, Brezillon S, Tyldesley R, Blanpain C, Detheux M, Mantovani A, Sozzani S, Vassart G, Parmentier M, Communi D (2003) Specific recruitment of antigen-presenting cells by chemerin, a novel processed ligand from human inflammatory fluids. J Exp Med 198:977985

Yazawa H, Yu ZX, Le Takeda Y, Gong W, Ferrans VJ, Oppenheim JJ, Li CC, Wang JM (2001) Beta amyloid peptide (A $\beta 42)$ is internalized via the G-protein-coupled receptor FPRL1 and forms fibrillar aggregates in macrophages. FASEB J 15:2454-2462 